\title{
Voltage-Dependent Gating of Single Sodium Channels from Mammalian Neuroblastoma Cells
}

\author{
Richard W. Aldricha and Charles F. Stevens \\ Section of Molecular Neurobiology, Yale University School of Medicine, New Haven, Connecticut 06510
}

Single sodium channel currents have been studied in cellattached patches from the mouse neuroblastoma cell line N1E115. Distributions of open duration, latency until first opening, and the average probability of a channel being open after a voltage step, $p(t)$, were analyzed and compared to predicted distributions from various kinetic models for voltage-dependent gating. It was found that, over most of the voltage range under which channel gating occurs, the slow steps in gating are opening transitions and that inactivation of open channels is significantly faster than the decline in $p(t)\left(\tau_{h}\right)$. This view of gating is confirmed by comparison of the kinetics of ensemble averages of single-channel currents obtained from step- and tail-current records at the same voltage. The probability of a channel reopening after having closed was calculated by comparing $p(t)$ with the convolution of the first-latency probability density and the conditional probability of remaining open $t$ milliseconds after opening. This reopening probability is small but slightly voltage dependent over the voltage range where the mean open duration remains constant and $\tau_{h}$ changes considerably. The voltage dependence of open channel inactivation and deactivation were calculated from the probability of reopening and the mean open duration. The equivalent gating charge for the inactivation rate is a few tenths of an electronic charge, whereas the equivalent charge for the closing rate is 2.5-3.5 electronic charges.

Unraveling the molecular mechanisms of nerve excitation will require precise knowledge of the states the sodium channel can occupy and transition rates between these states. We have used single-channel recordings to develop a description of a small part of the sodium channel's behavior, specifically to estimate the rates at which channels leave the open state.We shall conclude that channels have only a single kinetically defined open state, that the inactivation rate is significantly greater than the deactivation (closing but not inactivating) rate over a wide voltage range, and that the inactivation rate constant (from the open state) is only weakly voltage dependent, whereas deactivation

\footnotetext{
Received Jan. 4, 1986; revised July 7, 1986; accepted Aug. 21, 1986.

We wish to thank Charles $\mathrm{K}$. Solc for collaborating on some of the experiments (data given in Fig. 6) and for help with the preparation of the figures; and Denis Baylor for comments on the manuscript. This work was supported by National Institute of Health Grants NS23294 to R.W.A. and NS12961 to C.F.S. and a grant from The Chicago Community Trust/Searle Scholar's Program to R.W.A.

Correspondence should be addressed to C. F. Stevens, Section of Molecular Neurobiology, Yale University School of Medicine, 333 Cedar Street, New Haven, CT 06510.

2 Present address: Department of Neurobiology, Stanford University School of Medicine, Stanford, CA 94305.

Copyright (C) 1987 Society for Neuroscience $0270-6474 / 87 / 020418-14 \$ 02.00 / 0$
}

depends quite strongly on voltage. After a step increase in membrane potential, the time course of current flow is, under many circumstances, dominated by channel opening rather than some other process (such as inactivation or simple channel closing).

The results reported here, including an analysis of channel behavior during tail currents, confirm and extend our earlier observations (Aldrich and Stevens, 1983; Aldrich et al., 1983). Perhaps our most striking finding is that activation processes, rather than inactivation, dominate macroscopic sodium channel kinetics over a surprisingly large voltage range. What has traditionally been recognized as the kinetics of inactivation, then, appears to be a manifestation of a slow component of the activation process in the type of sodium channels we have studied.

\section{Materials and Methods}

General methods. The data reported here were obtained with the singlechannel recording method from cell-attached patches on cultured neuroblastoma cells of the type N1E115. The cells were maintained in Delbecco's modified Eagle's medium plus $5 \%$ fetal calf serum and grown at $37^{\circ} \mathrm{C}$ in a $5 \% \mathrm{CO}_{2}$ atmosphere. They were plated onto glass coverslips in $35 \mathrm{ml}$ tissue culture dishes. Cells were used for patch-clamp experiments from $1 \mathrm{~d}$ to 2 weeks after plating. During this time the density of sodium channels (as judged by the number of channels per patch) increased. In some cases, the cells were plated into medium containing $10 \%$ fetal calf serum. Cells in medium containing higher serum were slower to differentiate and consequently had lower sodium channel densities during the first week after plating. No differences in sodium channel behavior were seen between cells in high- and low-serum media. Pipette and hath solutions contained mammalian Ringer's solution (1 50 mM NaCl, $5 \mathrm{~mm} \mathrm{KCl}, 4 \mathrm{~mm} \mathrm{MgCl}_{2}, 2 \mathrm{~mm} \mathrm{CaCl}_{2}, 10 \mathrm{~mm}$ HEPES, pH 7.2). Because we have mostly analyzed data from cell attached patches, the absolute value of the membrane potential is unknown. All potentials are reported relative to the resting membrane potential. Holding potential was maintained 60 or $70 \mathrm{mV}$ more negative than the resting values. Test voltages were preceeded by a 300 -msec-long step to very negative voltages to remove inactivation: Prepulses to $-120,-140$, and $-160 \mathrm{mV}$ (relative to rest) were equally effective in removing inactivation. Ensembles of 64 current records were collected with a given voltage pulse sequence. The pulse sequences were repeated once every 800 msec.

Current records were filtered with an 8 pole Bessel filter with a cutoff frequency of $2500 \mathrm{~Hz}$ and sampled once per $100 \mathrm{msec}$. Leak and capacitative current subtraction was achieved hy subtracting a scaled average of 32 current records obtained during steps to a voltage at which no channel openings occurred. Changes in the time course of ensemble averages at a given voltage were taken as signs of experimental drift. Segments of data were analyzed only if they were free of drift. Temperature usually was $11^{\circ} \mathrm{C}$, but other temperatures have been used as specified in the text.

Missed events. The filter characteristics are such that the dwell time at half-maximum response is, in the noise-free case, equal to the open channel lifetime; for that reason, all open durations have been measured at the criterion level of $0.5 i_{u}$, where $i_{u}$ is the single-channel current (see Colquhoun and Sigworth, 1983).

When durations are measured in this way, 5 sorts of errors occur. (1) Some events are so brief that the filter response does not reach the 
criterion level. This effect gives rise to missed events and makes the estimate of the mean open time longer than it should be because shorter events are systematically excluded. (2) If the noise level of the recording amplifier and electrode is large enough, noise crossings of the criterion level will occasionally be interpreted as openings. This effect causes open times to be underestimated because the shortest bins in the open time histogram will contain more entries than they should. (3) If the underlying event was too brief to give a full-sized filter response, the dwell time above criterion will be less than the duration of the underlying opening. (4) Fluctuations in current due to noise in the recording system can cross the criterion, leading to over- or underestimates of the duration of the underlying event. (5) The fact that the sampling interval is comparable to some short open durations gives rise to a "scattering error" in which events that should be entered in 1 bin of the histogram in fact appear in a neighboring bin. For example, if the sample interval is 100 $\mu \mathrm{sec}$, an event with a duration of $150 \mu \mathrm{sec}$ (which should appear in the $100-200 \mu \mathrm{sec}$ bin) will be recorded half of the time as $100 \mu \mathrm{sec}$ long (exceeds criterion for 1 sample point) and half of the time as $200 \mu \mathrm{sec}$ long (exceeds criterion for $200 \mu \mathrm{sec}$ ).

We have estimated the magnitude of these errors by simulations in which square currents of the appropriate amplitude and with various durations were added to noise recorded during experiments under conditions that did not produce sodium channel openings. The simulated openings were then filtered and analyzed with the same computer programs used for our experimental records. Dwell time histograms were corrected according to these empirical results. The various effects do not, under the conditions of our experiments, produce appreciable errors in our estimates of mean open time or single-channel current.

\section{Theory}

The goal of this section is to develop an equation that permits us to estimate the probability that a channel, once open, will reopen after closing. The final result is

$$
R=1-(1-Q) D / I
$$

where $R$ is the probability of reopening, $Q$ is the probability that a channel passes from the resting state into the inactivated state without ever opening, $D$ is the mean open duration, and $I$ is the integral of the function $p(t)$, the probability that a single channel will be open at time $t$ after the onset of a depolarizing pulse. Most of our patches have more than 1 channel. If $N$ is the number of functioning channels per patch, then $p(t)$ is the average current as a function of time divided by the quantity $N i$, where $i$ is the single-channel current. The probability $Q$ is estimated by taking the $N$ th root of the fraction of depolarization epochs in which no channel opens (see Appendix for terminology).

To explain this method for estimating the probability of reopenings, we must investigate the properties of 2 functions $p(t)$ and $\hat{p}(t)$. The first of these, the $p(t)$ defined above, is the probability that a channel will be found open $t \mathrm{msec}$ after the onset of depolarization; the second function, $\hat{p}(t)$, is the same probability for the special case of an identical channel that we suppose cannot reopen. The basic idea behind this method is that the reopening probabilities can be found by comparing $p(t)$ and $\hat{p}(t)$ in the way described below. It will be seen that $p(t)$ can be estimated directly and that $\hat{p}(t)$ can easily be calculated from experimentally measured quantities.

If $f(t)$ is the probability density for first openings (after the start of a depolarization epoch) and $m(t)$ is the conditional probability that a channel will be open $t$ msec after first opening, then $p(t)$ is given by the convolution of $f$ and $m$ (Aldrich et al., 1983):

$$
p(t)=f(t) * m(t) .
$$

If each channel may open only once per depolarization epoch, then $\hat{p}(t)$, the value of $p(t)$ for this special case, is given by

$$
\hat{p}(t)=f(t) * w(t)
$$

where $w(t)$ is the conditional probability that a channel is still open $t$ msec after first opening without having closed. Whenever reopenings occur, $p(t)$ will be greater than $\hat{p}(t)$, and the probability of reopenings can be estimated from the extent to which $p(t)$ exceeds $\hat{p}(t) ; p(t)$ can be estimated directly from singlechannel current averages and $\hat{p}(t)$ can be calculated from first latencies and the distribution of dwell time in the open state by using Eq. (3).

We have shown previously that Eq. (3) for $\hat{p}(t)$ is actually a good approximation to the observed $p(t)$ under at least some circumstances, but we have not quantified the significance of any deviations between predictions and observations. Our goal, then, is to estimate the reopening probability from the discrepancy between the $\hat{p}(t)$ predicted by Eq. (3) and the experimentally observed $p(t)$. It will turn out that it is more convenient to work with the time integrals of $p$ and $p$ than with the functions themselves.

If we integrate both sides of Eq. (2) from 0 (the start of the depolarization epoch) to infinite time, the result is

$$
I=(1-Q) M
$$

where $I$ is the integral of $p(t), M$ the integral of $m(t)$, and (1 $Q$ ) is the integral of the probability density for first openings; $Q$ is the probability that a channel never opens (because it enters the inactivated state without opening), so that $(1-Q)$ is the probability that a channel opens 1 or more times during the depolarization epoch. This manipulation makes use of the causality property of $m(t)$; that is, $m(t)$ is zero for negative arguments. We shall employ causality several times in later steps of our derivation.

We now must examine $m(t)$ and $M$ more carefully. If a channel is found open $t$ msec after first opening, either it never closed or it closed and reopened. Therefore,

$$
m(t)=w(t)-g(t) * \frac{d w}{d t}
$$

where $w(t)$ is the probability that a channel is open at $t$ without having closed after first opening, $-d w / d t$ is the probability density for closing for the first time at $t$, and $g(t)$ is the probability that a channel is open $t$ msec after first closing.

On integrating this equation from 0 to infinity, we obtain

$$
M=D+G
$$

where $D$ is the mean dwell time in the open state and $G$ is the integral of $g(t)$, the contribution of reopenings to $M$. This relationship is correct because $w(t)$ is found experimentally to obey the equation

$$
w(t)=\exp (-t / D)
$$

Now we examine $g(t)$ and its integral $G$. If the open state is unique, that is, if an open channel is in the same state irrespective of whether it has opened one or more times, then

$$
g(t)=r(t) * m(t)
$$

where $g(t)$ and $m(t)$ are as defined above, and $r(t)$ is the probability density for first reopening $t \mathrm{msec}$ after closing. If we integrate both sides of this equation over the entire depolarization epoch, then 


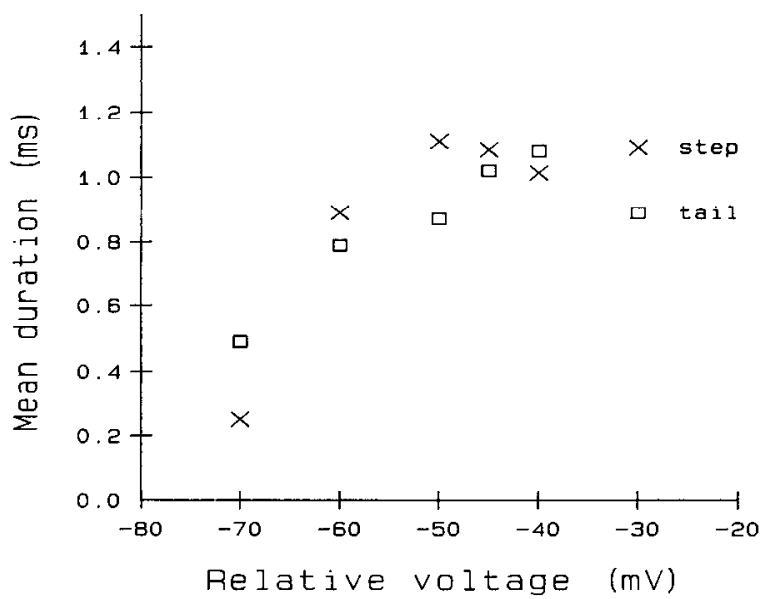

Figure 1. Comparison of mean open durations during voltage steps to a particular voltage (step opening) and at the same voltage after a conditioning pulse to $+20 \mathrm{mV}$. (tail openings). Voltages are relative to the (unknown) resting potential of the cell.

$$
G=R M
$$

where $R$ is the integral of $r(t)$ and gives the probability of a channel sometime reopening after having left the open state. Therefore, the pair of equations $M=D+G$ and $G=R M$ give, when $G$ is eliminated, the relation

$$
M=D /(1-R)
$$

because

$$
\begin{aligned}
& I=(1-Q) M \\
& I=(1-Q) D /(1-R) .
\end{aligned}
$$

This may be rearranged to give the final result

$$
R=1-(1-Q) D / I
$$

Note that the integral of $\hat{p}(t)$ is $(1-Q) D$, so that $R$ is found from the ratio of the integrals of $p$ and $\hat{p}$.

The reopening probability depends on 2 factors: the probability $1-F$ (where $F$ is the probability of an open channel inactivating) that a channel makes a transition from the open to the closed state rather than the inactivated state, and the probability $1-Q$ that a closed channel makes a transition from closed to open rather than closed to inactivated; here we assume that the probability for inactivation is effectivcly the same for all closed states, either inherently or because these states are in rapid equilibrium on the time scale of closed channel inacti-

\section{Table 1. Test of open-channel independence}

\begin{tabular}{lrlll}
\multicolumn{2}{l}{ Patch $V_{c}$} & Level 1 & Level 2 & Ratio \\
\hline 26 & 20 & $0.71 \pm 0.03(874)$ & $0.38 \pm 0.04(199)$ & 1.87 \\
26 & 10 & $0.72 \pm 0.03(720)$ & $0.32 \pm 0.06(103)$ & 2.25 \\
32 & -20 & $0.73 \pm 0.02(2378)$ & $0.38 \pm 0.03(517)$ & 1.91
\end{tabular}

Command voltage $\left(V_{c}\right)$ is specified in millivolts displacement from the resting potential. Holding potentials for patch 26 ranged from -40 to $-80 \mathrm{mV}$ for different runs, and for patch 32 from -80 to $-140 \mathrm{mV}$. Level $n$ gives the mean dwell time \pm SEM (both in msec) followed (in parentheses) by the number of observations for $n$ channels open. None of the mean open times are significantly different from one another. Ratio gives the mean level 1 divided by the mean level 2 . None of the ratios is significantly different from the value 2 expected from the assumption of independence.
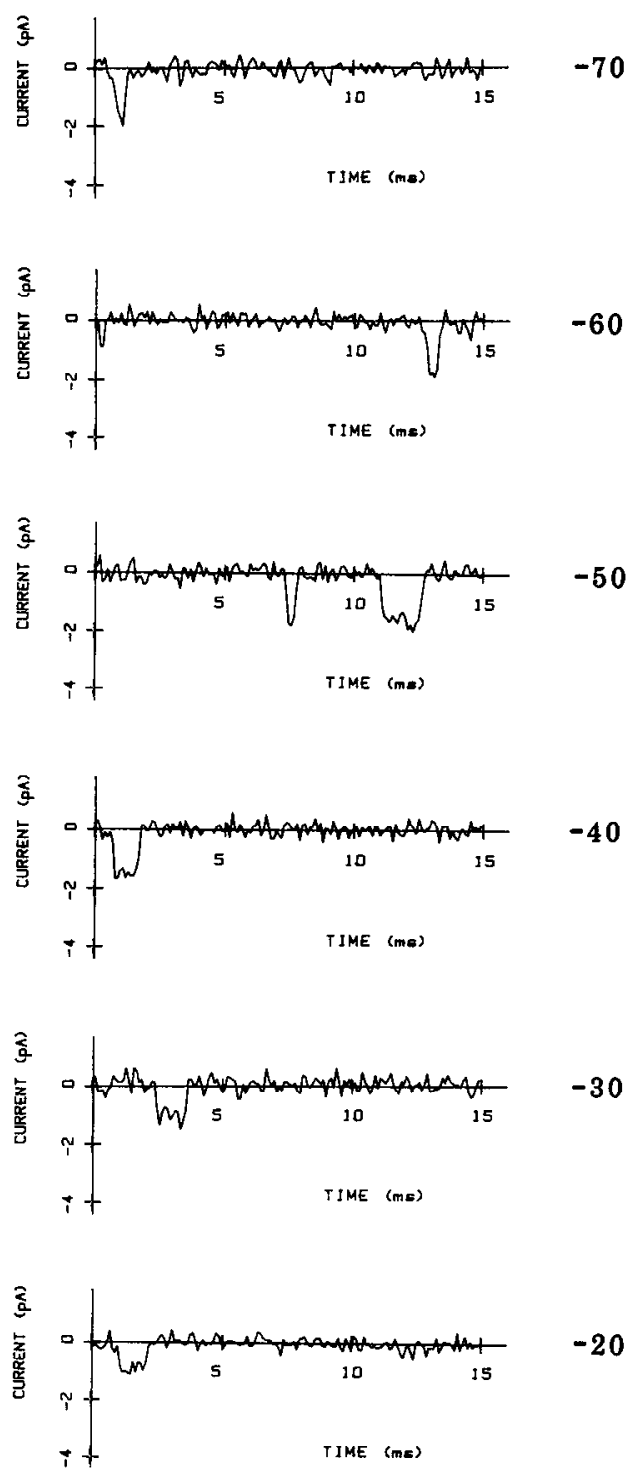

Figure 2. Representative openings at voltages between -70 and -20 $\mathrm{mV}$ (relative to resting potential). Leakage and capacitative current have been digitally subtracted (see Materials and Methods). The single-channel current amplitudes are approximately $1 \mathrm{pA}$ at -20 and saturate at approximately $2 \mathrm{pA}$ at -70 , equivalent to a single-channel conductance of about $30 \mathrm{pS}$ over the linear range of the current-voltage relationship. Data were filtered with an 8 pole Bessel filter with a cutoff frequency of $2500 \mathrm{~Hz}$ and sampled once per $100 \mathrm{msec}$. Temperature was $11^{\circ} \mathrm{C}$.

vation. Thus,

$$
R=(1-F)(1-Q) \text {. }
$$

We rearrange this equation to obtain an equation for $F$ (probability of open to inactivated transition) in terms of $R$ (reopening probability) and $Q$ (probability of resting to inactivated transition):

$$
F=1-R /(1-Q) \text {. }
$$

Because $(1-Q)$ may underestimate the opening probability of a channel that has just closed (and is thus very close to the open state), $F$ in this equation is a lower limit for the true probability of an open channel passing to the inactivated state. That is, the 

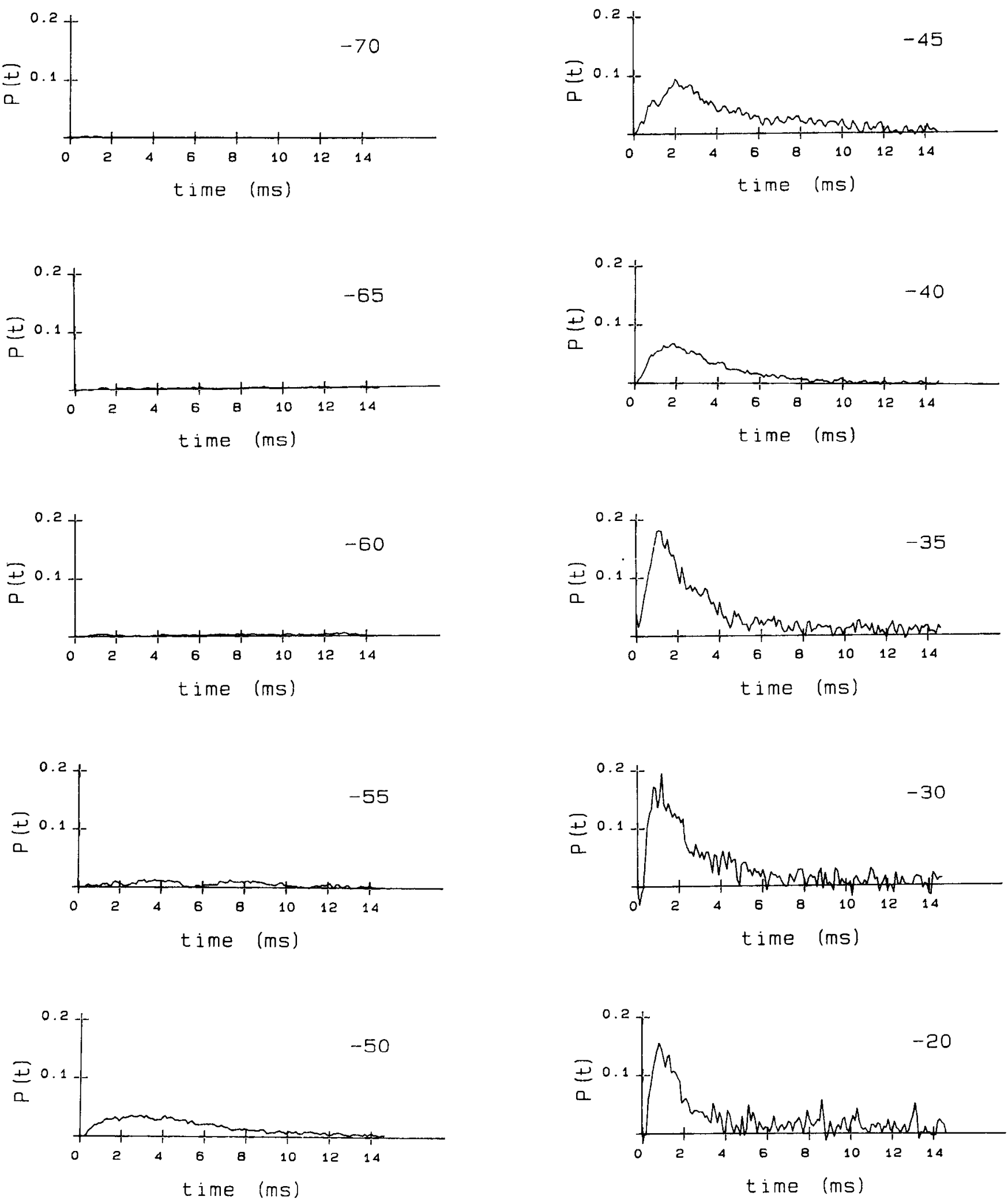

Figure 3. Ensemble averages of single-channel openings over the voltage range -70 to $-20 \mathrm{mV}$. Inactivation was removed by a $300 \mathrm{msec}$ conditioning prepulse to $-120,-140$, or $-160 \mathrm{mV}$ before the step to the indicated voltage. Sixty-four to 1200 single-channel records were averaged at each voltage to obtain the time-dependent probability of a channel being open after a voltage step $p(t)$. $P(t)$ was calculated according to the equation $P(t)=I(t) /(N i)$, where $I(t)$ is the ensemble average current, $N=$ the number of channels in the patch (4), and $i=$ the single-channel current at the given voltage. 

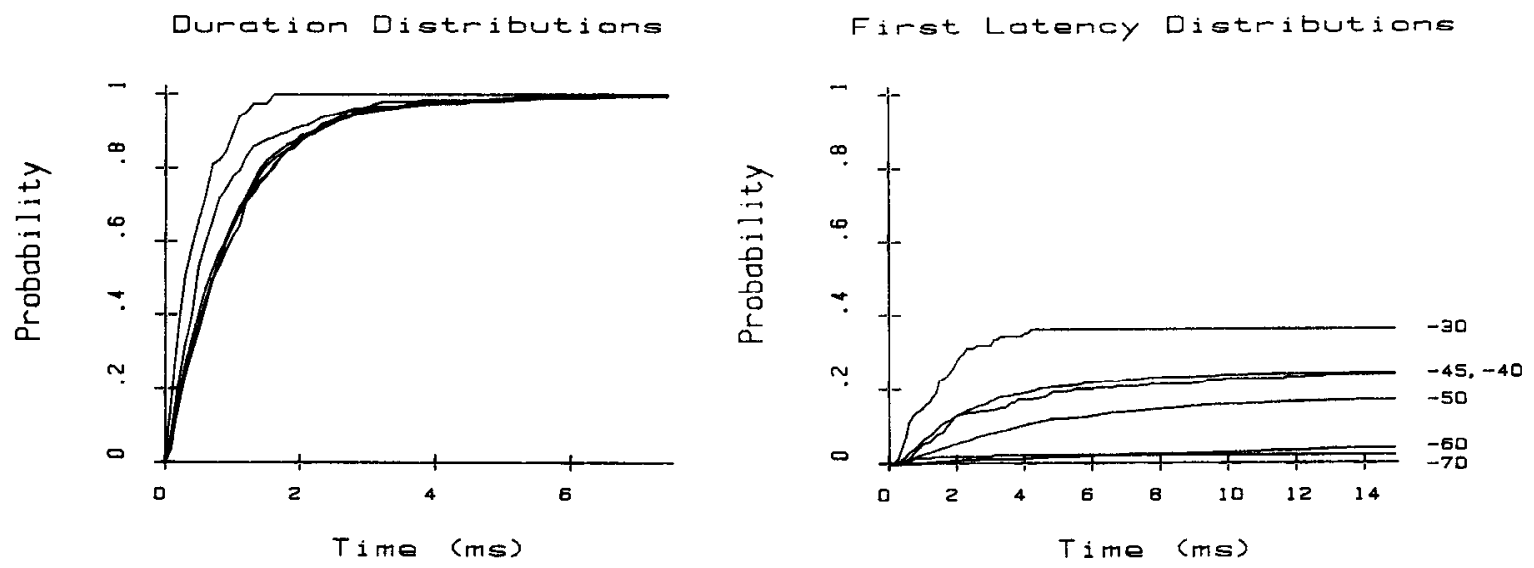

Figure 4. Voltage dependence of open time and first-latency distributions. Right-hand panel, Cumulative duration distributions recorded at -70 , $-60,-50,-40,-30 \mathrm{mV}$. Resting inactivation was varied by using appropriate prepulse voltages such that only single openings occurred. Because of this, no correction for overlapping events is necessary. The distributions can be well fitted by single-exponential functions (Kolmogorov-Smirnov test, $5 \%$ level). Open times are shorter at -70 and $-60 \mathrm{mV}$, but there is no voltage dependence in the distributions at higher voltages. Right-hand panel, Cumulative first-latency distributions for the corresponding voltages. These functions plot the probability of observing a first opening less than $t$ milliseconds after a voltage step. They have been corrected for the number of channels in the patch (4) (see Patlak and Horn, 1982). Inactivation was removed with a $300 \mathrm{msec}$ prepulse to -120 or $-140 \mathrm{mV}$. The distributions have asymptotic values less than one due to the significant probability of observing a record in which no openings occur due to inactivation of closed channels.

correct value for $F$ satisfies the inequality

$$
F<1-R /(1-Q)
$$

\section{Results}

Our overall conclusions are based on observations made on membrane patches from 103 cells; of these, 70 patches were cell attached, 23 were inside-out, and 23 were outside-out (in some cases the same patch was both cell attached and inside- or outside-out). Because we find that channels in non-cell-attached patches have steady-state inactivation that is shifted significantly in an hyperpolarizing direction, have much longer open times, and tend to change their behavior over the course of an experiment, the analysis here is limited to recordings made in the cell-attached configuration. Our specific conclusions are illustrated by data obtained from a single patch containing 4 channels and are supported by analysis of data from other patches as indicated below.

\section{Open channels close independently}

An important assumption for all analyses of sodium channel currents is that channels are independent of one another. Because our goal here has been to examine transition rates out of the open state, we have investigated the extent to which open channels behave independently, and we have also studied the effect neighboring closed or inactivated channels have on rates of leaving the open state. We have been unable to detect channelchannel interactions and conclude that possible interchannel cooperative effects do not play an appreciable role in the phenomena we have investigated.

Evidencc of interchanncl coopcrativity has becn sought in 2 ways. First, we have compared the length of time 2 channels remain simultaneously open with what would be predicted from the open lifetime of a single channel. Second, we have altered the average initial state of channels in the patch with a large depolarizing prepulse and compared the behavior with that of channels in a patch not subjected to a prepulse; the idea here is to compare the behavior of an open channel in patches where neighboring channels are in very different states.
If a single channel leaves a unique open state with a total rate (for deactivation and inactivation combined) $a$ and if 2 open channels do not cooperate, then 2 simultaneously open channels should leave the (doubly) open state with a rate $2 a$. A singly open channel, then, would remain in the open state an average of $t=1 / a \mathrm{msec}$, and a pair of open channels would both remain open $1 / 2 a=t / 2$ msec. Similarly, 3 channels should remain simultaneously open for $1 / 3 a \mathrm{msec}$, etc; levels greater than 2 are subject to successively greater errors because very short openings are undetected and this leads to overestimates of the average dwell time in the multiply open state. Table 1 displays the average open time for both singly and doubly open channels. The residence time at the doubly open level is not significantly different from what the singly open dwell time would predict, an observation consistent with the independence of open channel behavior.

Open channel dwell times described above were obtained after the patch was subjected to a moderate step depolarization. If the patch is strongly depolarized briefly, channels will open or inactivate with a high probability and will tend to be in a different state on return to a less strong depolarization; these are the conditions for recording "tail currents." We have investigated sodium channel behavior during tail currents to determine if neighboring channels with different histories (step vs prepulse), and consequently different average states, differentially affect the behavior of the specific channel being observed. Figure 1 shows that the average open time after a brief, large prepulse to $+20 \mathrm{mV}$ (relative to the resting potential of the cell) is the same as that during a moderate step depolarization to the same voltage level in the range -70 to $-30 \mathrm{mV}$. The state of channels in a patch appcars, then, to have no significant cffect on the behavior of open neighbors, at least under the conditions of these experiments. Horn et al. (1984) have reached a similar conclusion for channels that have been modified by treating them with $N$-bromoacetamide.

\section{Channel behavior over a range of voltages}

Figures 2-4 illustrate sodium channel behavior over a $50 \mathrm{mV}$ range of depolarizations that span the major part of the sodium 


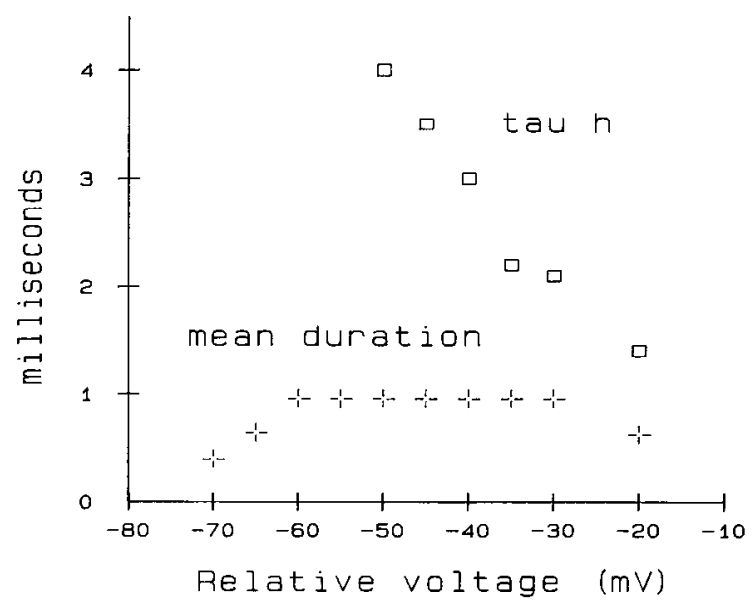

Figure 5. Voltage dependence of mean open duration and $\tau_{h}$. Mean durations were obtained from exponential fits of duration distributions such as those in Figure 4. $\tau_{h}$ was measured by a single-exponential fit to the decline of average current records such as those in Figure 3.

channel's activation voltages. Figure 2 presents patch current flow during individual depolarization epochs and provides illustrations of the typical signal-to-noise ratios. The single-channel conductance derived from such records is $30 \mathrm{pS}$. The current-voltage relationship saturates below $-60 \mathrm{mV}$ at about 2 pA. Figure 3 displays the probability of a channel being open after a voltage step for the voltage range encompassed. These curves were obtained by averaging many records such as those shown in Figure 2 at each voltage and dividing the averages by the number of channels in the patch (4) and the single-channel current at the given voltage. These averages indicate that at the smallest depolarizations little activation occurred and that the kinetics of $p(t)$ become faster as the voltage increases. The averages are typical of those found in a variety of preparations.

In Figure 4 the cumulative histograms for dwell time in the open state and the time to first opening are presented. Depolarizing prepulses were used to eliminate most of the channels in the patch. This procedure reduced channel openings considerably and inactivated simultaneous openings. These open time cumulative histograms are not significantly different from single-exponential distribution functions (Kolmogorov-Smirnov test, $5 \%$ level), a consistent observation in all of our cell-attached patches. For most voltages the duration distributions are indistinguishable, but for the smallest depolarizations $(-70$ and -60 in Fig. 4), the channel dwells in the open state for a shorter time.

Whereas the open state dwell time distributions are not significantly voltage dependent over most of the activation range, this is not true for the time to first opening after a depolarization: The first-latency distributions vary appreciably with voltage over the entire depolarization range. The voltage dependence of macroscopic kinetics thus resides mostly in the steps leading to opening, and not in the open state except at the smallest depolarizations.

Mean channel open time as a function of voltage is presented in Figure 5; the same figure also illustrates $\tau_{h}$ as a function of voltage. Note that $\tau_{h}$ is always much longer than the mean open time except for the largest depolarization, where $\tau_{h}$ approaches its shortest values. Figure 6 shows mean open dwell time plotted against $\tau_{h}$ for 3 different patches. These plots have axes with equal time increments to facilitate comparison of the mean duration with $\tau_{h}$. They are comparable to Figure 5 of Aldrich
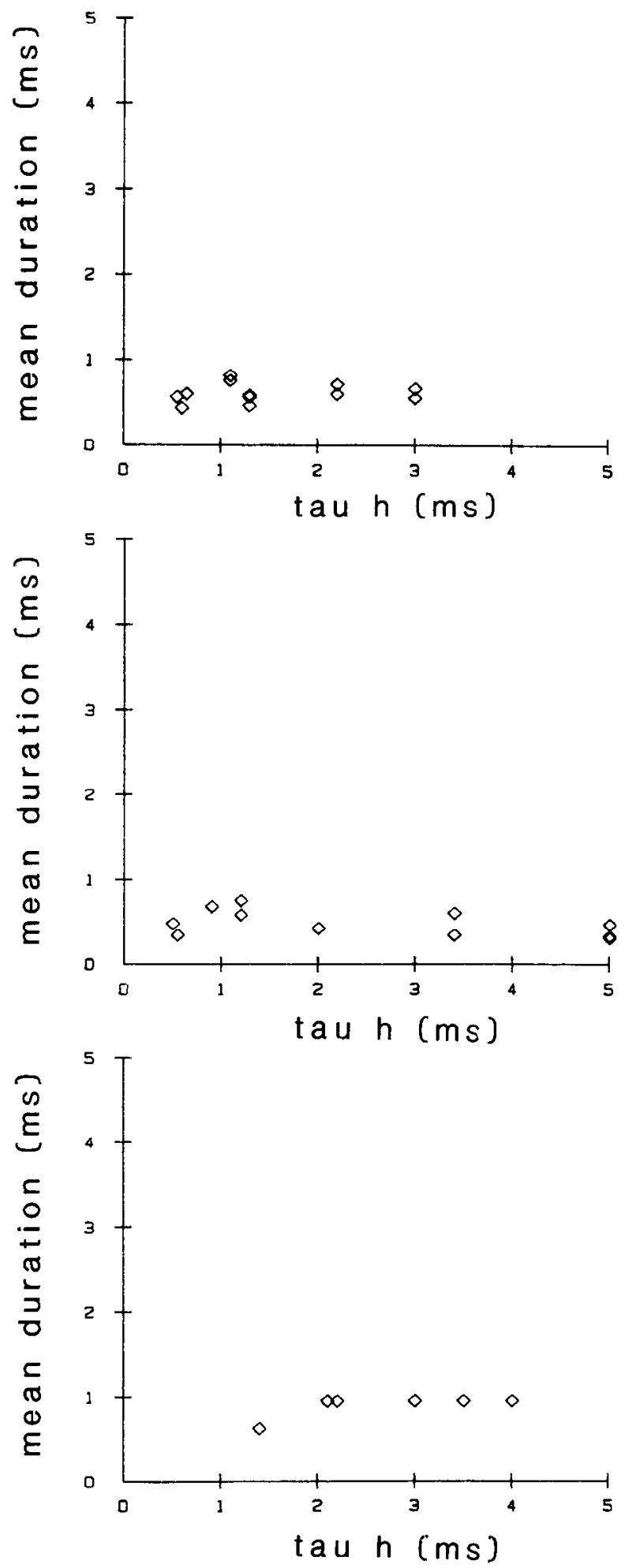

Figure 6. Comparison of mean duration and $\tau_{h}$ in 3 different patches. Each graph plots mean duration versus the value of $\tau_{h}$ obtained from average currents at the same voltage. The abscissa corresponds nonlinearly to inverse voltage with higher voltages (where $\tau_{h}$ is smaller) nearer the origin. In each case, mean open durations are much less voltage dependent than $\tau_{h}$. They become equal only at high voltages where the overall kinetics are fast. The bottom panel contains data from the same patch as the rest of the figures in the paper. Temperatures: $t o p, 17^{\circ} \mathrm{C}$; middle, $15^{\circ} \mathrm{C}$; bottom, $11^{\circ} \mathrm{C}$. 

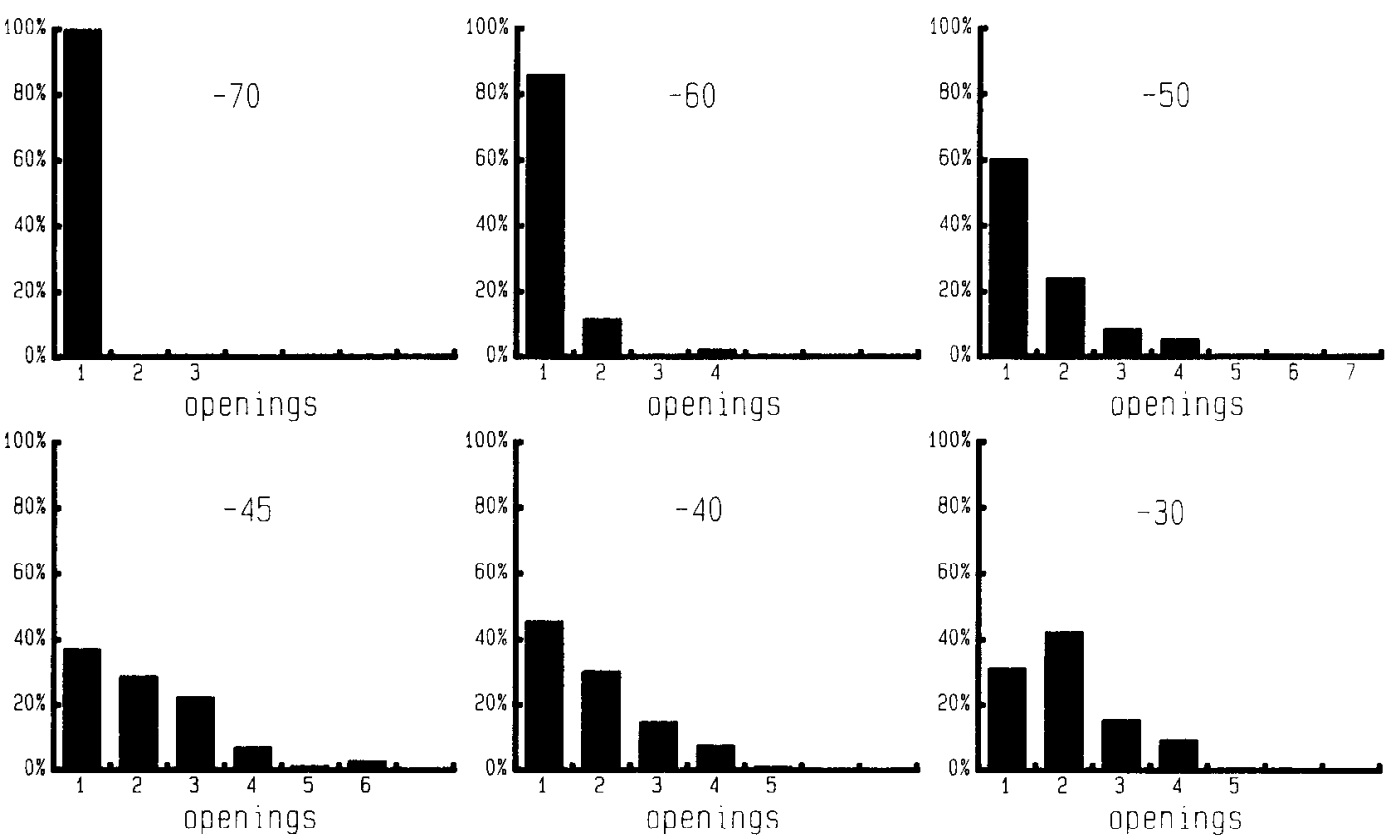

Figure 7. Voltage dependence of the number of openings per voltage step. Each panel shows a distribution of the number of openings per voltage step given that at least 1 opening occurred. Blank records are not included. The patch contained 4 channels. Records with greater than 1 opening per channel (which must result from reopenings of a channel that has closed) rarely occur.

et al. (1983) except that they show data obtained from single patches as opposed to pooled data from a number of patches. In all cases the open duration is much less than $\tau_{h}$ and varies much less with voltage. We have given evidence elsewhere that open channels usually inactivate rather than deactivate for most of the activation range of voltages (Aldrich and Stevens, 1983; Aldrich et al., 1983). These figures illustrate our previous conclusion that $\tau_{h}$ does not represent the time constant for open channel inactivation except at the largest depolarizations.
A channel generally opens only once per depolarization epoch Figure 7 shows histograms of the number of openings per depolarization epoch for our 4 channel patch over the same voltage range as the duration and latency distributions of Figure 4 . These histograms include both single isolated openings and simultaneous multiple openings. If each channel opened a single time during the voltage step, there would never be more openings than the number of channels in the patch (4). The histograms

Figure 8. Comparison of $p(t)$ and $\hat{p}(t)$. $p(t)$ is the probability of a channel being open after a step to the indicated voltage taken from ensemble averages of single-channel records. $\hat{p}(t)$ is the convolution of the first-latency probability density function $f(t)$ and the conditional probability that a channel is still open $t$ msec after first opening without having closed, $w(t)$, according to Eq. (3). The convolution was performed by multiplying the Fourier transforms of the respective functions.
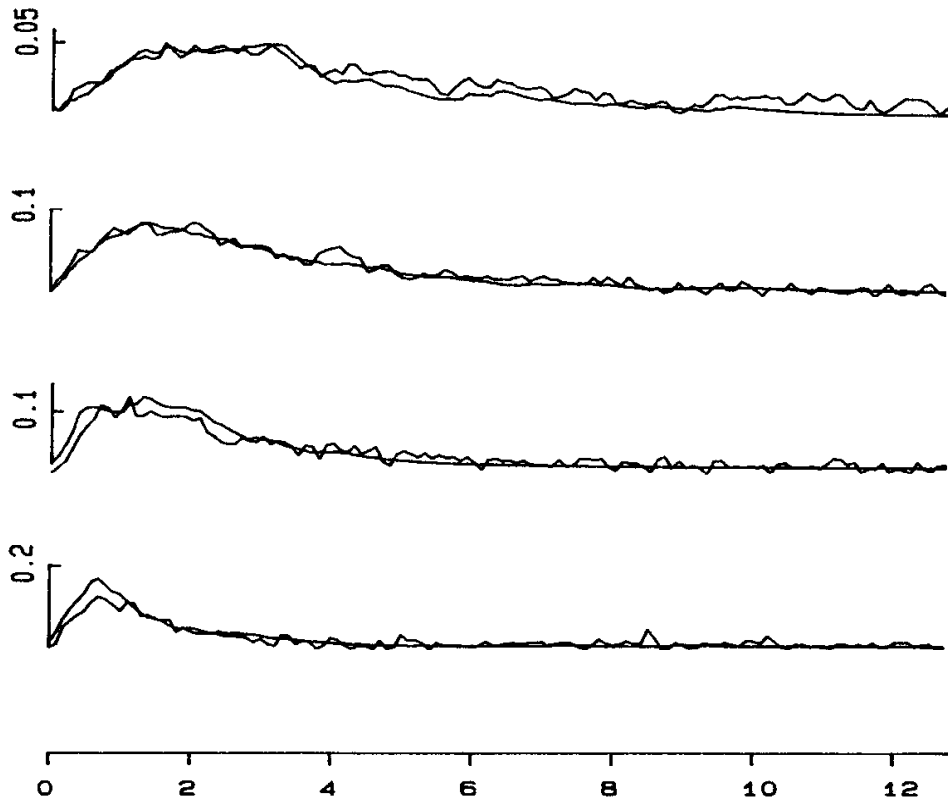

time (ms) 


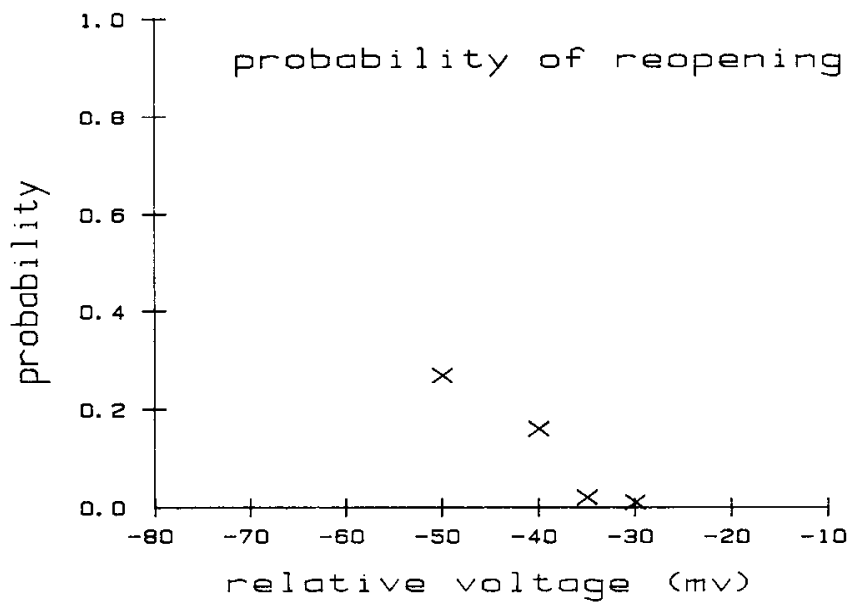

Figure 9. Voltage dependence of the probability of a channel reopening after having closed. Values for reopening probabilities were calculated by comparing integrals of $p(t)$ and $\hat{p}(t)$ [Eq. (1)].

show that there are rarely more than 4 openings over the entire voltage range, although more than 4 openings do occur during the $15 \mathrm{msec}$ depolarization (more than 4 channels are never simultaneously open). Greater than 4 openings implies a nonzero probability of channels reopening after closing. We have previously analyzed such histograms to calculate the fraction of openings that close to the inactivated state (Aldrich and Stevens, 1983; Aldrich et al., 1983). The present data were not satisfactory for such analysis because of the high percentage of records with no openings. This caused a wide range of suitable fits for the data. We have therefore used a different method (outlined under Theory) to calculate the fraction of openings that close by inactivating. If a channel opens only once per depolarization epoch, the sodium current [or probability of a channel being open as a function of time, $p(t)]$ should be accurately predicted by the convolution of the first latency density function and the open time distribution function (Fig. 4). The results of these convolution operations are shown in Figure 8 superimposed on the observed $p(t)$ functions. The predicted and observed curves are obviously in good general agreement.

As indicated in the Theory section, the ratio of the integral of the observed $p(t)$ and the $\hat{p}(t)$ predicted from the convolutions illustrated in Figure 8 provides, through Eq. (1), an estimate of the probability that a channel will reopen. Reopening probabilities as a function of voltage (relative to resting potential) are shown in Figure 9, which indicates that channels usually open only once per depolarization epoch with a maximum reopening probability of about 0.27 at the lowest depolarization.

\section{Estimates of rate constants for closing and inactivation from the open state}

Figure 5 presents mean open time as a function of membrane potential. Dwell time in the open state shows little voltage dependence for larger depolarizations - voltages greater than -60 $\mathrm{mV}$-but becomes progressively shorter at more hyperpolarized levels. We turn now to an analysis of these data to obtain estimates of the rates for channel deactivation and inactivation.

The average lifetime, $D$, of the open state is given by

$$
D=1 /(a+b)
$$

where $a$ is the transition rate from open to inactivated and $b$ is the deactivation (open to closed) rate. The probability that an

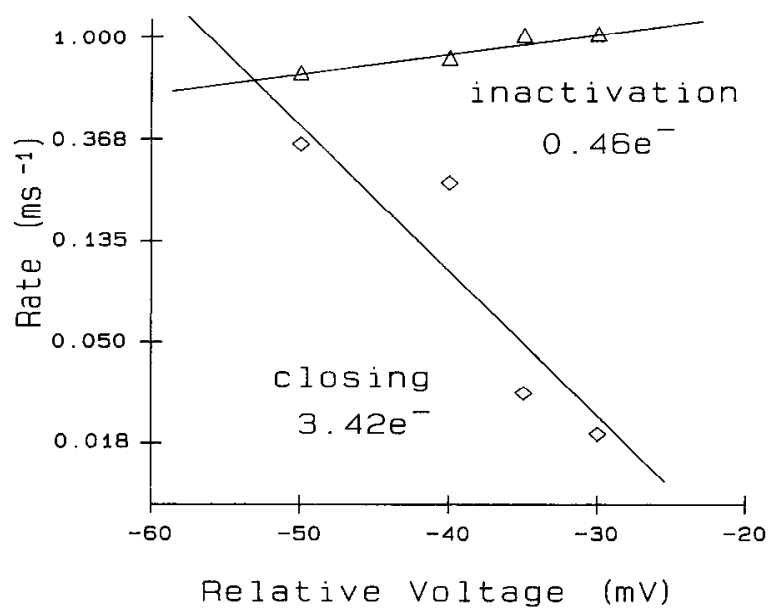

Figure 10. Voltage dependence of open channel inactivation and closing rates. Rate constants ( $a$ and $b$ ) are calculated from Eqs. (4a), (5), and (6) and plotted against voltage on a $\log$ (base $e$ ) axis. The values at various voltages are fitted with single-exponential functions, yielding equivalent gating charges of 0.46 for inactivation and 3.42 for closing (deactivation).

open channel will choose the inactivated rather than closed state when it makes a transition is then

$$
F=a /(a+b) \text {. }
$$

Equations (5) and (6) then provide the means for calculating $a$ (inactivation) and $b$ (deactivation), at different voltages, from the 2 experimentally obtainable quantities $D$ and $F$ [from Eq. (4a)]. We have used these equations in 2 different but related ways to estimate $a$ and $b$ as a function of membrane potential. The first way directly uses Eqs. (5) and (6), together with experimentally determined estimates of $D$ and $F$. The second way supposes that $a$ and $b$ depend exponentially on voltage and makes use of only qualitative properties of $F$, namely, that it is close to unity over a range of depolarized voltages.

With values of $D$ estimated from mean open times and $F$ obtained from Eq. (4a), we have used Eqs. (5) and (6) to derive $a$ and $b$ as illustrated in Figure 10, where rate constants are plotted semilogarithmically as a function of voltage. Straight lines on these graphs provide plausible fits to the data and yield an equivalent gating charge of 0.46 for inactivation and 3.42 for deactivation.

We turn now to the second method for estimating inactivation and deactivation rates. We know from estimates of the reopening probabilities (see Fig. 9) and the probability $Q$ of a closed channel passing to the inactivated state without even opening (see asympotic values of first-latency histograms in Fig. 4b) that, according to the inequality ( $4 b), F$ is approximately unity for the larger depolarizations (levels $>-40 \mathrm{mV}$ ). For these larger voltages, then, the mean open time directly gives an estimate of the open channel inactivation rate $a$. This observation confirms the conclusion reached earlier on the basis of a more detailed analysis using a somewhat different approach (Aldrich and Stevens, 1983; Aldrich et al., 1983).

For the small depolarizations the mean open time decreases. We suppose this decrease to reflect the deactivation rate which becomes large for more hyperpolarized voltages, so that $b$ dominates the mean open time for voltages below -60 in Figure 5 . Over the restricted voltage range represented in Figure 5, the inactivation and deactivation rate constants should depend approximately exponentially on membrane potential which means 

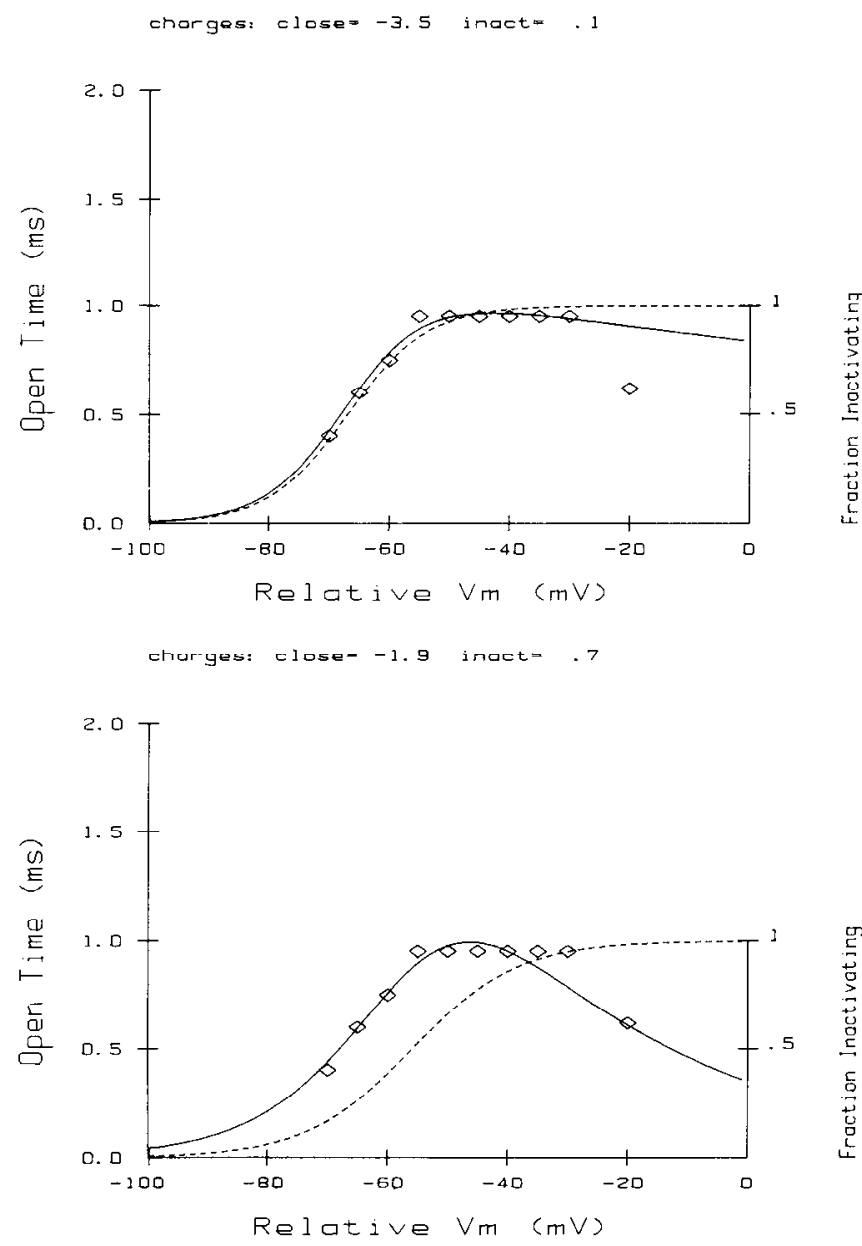

Figure 11. Range of acceptable values for the voltage dependence of open channel inactivation and deactivation. Mean open durations are plotted against voltage. The points are fitted assuming exponential voltage dependence of inactivation and deactivation rate constants by Eq. (7) (solid lines). The dashed lines plot the calculated probability of an open channel closing to an inactivated state (right-hand scale). Top and bottom panels show 2 alternate fits to the data that bracket the range of acceptable fits. Equivalent gating charges are $0.1-0.7 e^{-}$for inactivation and 1.9-3.5 $e^{-}$for closing.

that the mean open time $D$ should depend on membrane potential $V$ according to the equation

$$
D=1 /\left[a_{0} \exp \left(q_{a} V / k T\right)+b_{0} \exp \left(-q_{b} V / k T\right)\right]
$$

where $a_{0}$ and $b_{0}$ are the inactivation rates and deactivation rates at zero depolarization, $V$ is the membrane potential (relative to the cell resting potential), $q_{a}$ and $q_{b}$ are the charge movements associated with the respective transition states, $k$ is Boltzmann's constant, and $T$ is the absolute temperature.

The range of reasonable values for the equivalent changes of these transitions can be seen in Figure 11. Equation (7) has been fitted to the data in Figure 11 with various values for the 4 parameters $a_{0}, b_{0}, q_{a}$, and $q_{b}$. Because the open time data are subject to some error, plausible fits can be obtained over a range of values for the parameters. An additional constraint on the fits of theory to data is that $F=a /(a+b)$ should be close to unity, at least for depolarizations above $-40 \mathrm{mV}$ (values of $F$ for -40 and below are uncertain). With this additional constraint, $q_{a}$ must lie between 0.1 and 0.7 for the Figure 11 data, and $q_{b}$ must be in the range 3.5 and 1.9. In 5 other patches, $q_{a}$ was about 0.3 (with a possible range of $0-1$ ) and $q_{b}$ was about 3 (with a corresponding range of 2.5-3.4). We conclude, then, that the voltage sensitivity of the inactivation rate from the open state is equivalent to movement of about a third of an elementary charge and that deactivation is about 10 times more voltage sensitive.

\section{Analysis of channel behavior after a brief, large depolarization: tails}

Our previous analysis has shown that, over a range of activating voltages, a channel generally opens just once and then quickly inactivates. The time course of $p(t)$ for this voltage range, then, is dominated by the slow opening process, a slow component of activation, reflected in the first-latency histograms (Fig. 4b). We have further studied the relative contribution of opening and inactivation to the overall time course over most of the activation range by comparing the behavior of the channels after a step depolarization and following a repolarization from a conditioning depolarization (tail current).

An open channel whose function can be described as a timehomogeneous Markov process with a single open state should exhibit behavior at a given voltage that is independent of its past history of voltage or opening. Such a channel should have the same open duration and probability of inactivating irrespective of whether it opened during a depolarizing step to a given voltage or remained open after a step down from a higher voltage. We have compared the behavior of channels with different histories (with and without conditioning pulses) in an effort to detect departures from what would be expected of a time-homogeneous Markov system. We conclude that historic effects are not significant under the conditions of our experiments.

Figure 12 shows a comparison of these 2 types of experiment. The left-hand column show groups of sample records after depolarizing steps to a number of voltages. The right-hand column shows groups of records taken at the same voltages after a conditioning step to $+20 \mathrm{mV}$ (tail currents). The records in the lefthand column reveal the normal pattern of openings throughout the depolarization, with the latency of opening decreasing as depolarization is increased; the open durations increase with voltage at lower voltages and then remain constant with further depolarization (see Fig. 5). The openings after a large depolarizing prepulse (tail records) are, in contrast, grouped at the beginning of the trace, with the openings often superimposed. The open channels close quickly and only rarely reopen. The mean duration is not significantly different for the step and tail experiments at the same voltage (Fig. 1), consistent with there being only 1 open state and with an absence of cooperative effects. The tail records exhibit the behavior expected of channels that have opened synchronously: They are an empirical approximation of the conditional probability term $m(t)$ in Eq. (2). The contribution of opening kinetics to the overall time course is minimized, and the channels are seen to close and not reopen.

The conditional probability $m(t)$ of a channel being open at time $t$ after initially opening at time zero [see Eq. (2)] consists of 2 components, the duration term $w(t)$ that appears in Eq. (3) and a reopening term. If a channel has a significant probability of reopening, the second term will become large and the relaxation of the tail will be slower than the $w(t)$ process. Figure 13 compares average currents for both step and tail experiments at the same voltage, over the normal activation range. The 
Step
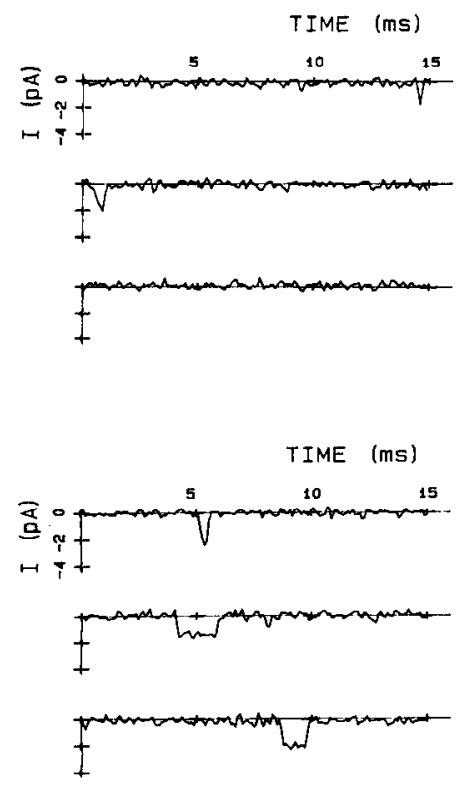

TIME (ms)

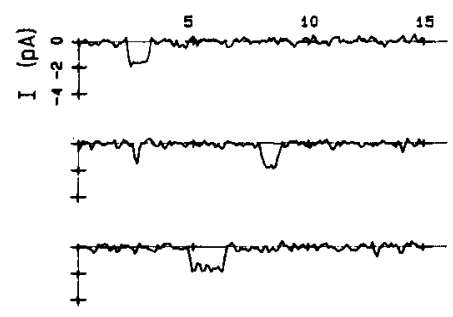

TIME (ms)
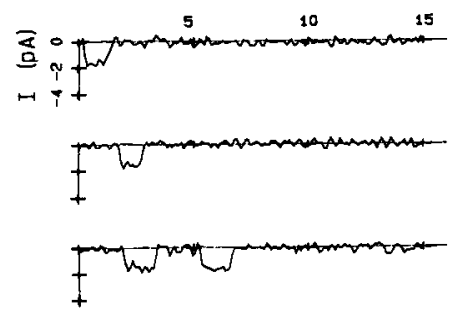

TIME (ms)
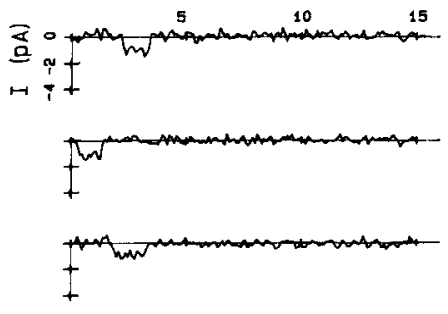

Tail
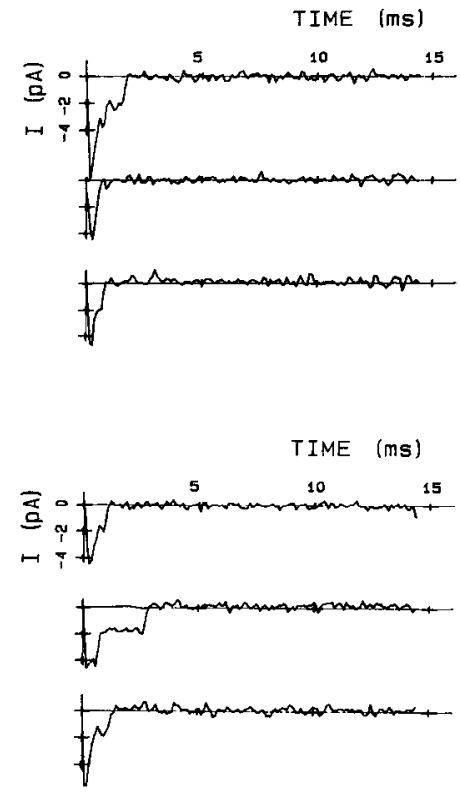

TIME (ms)

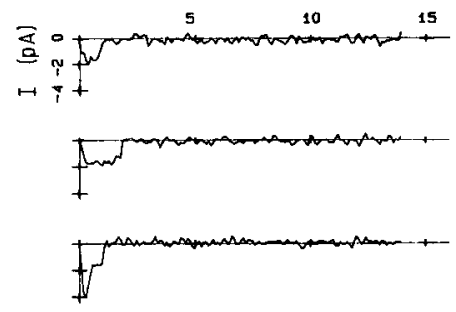

TIME (ms)
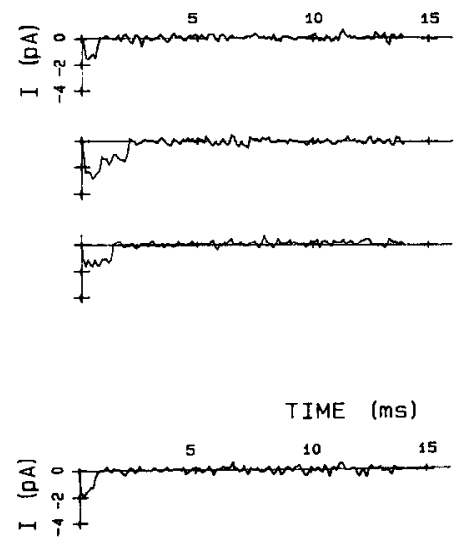

$-30$

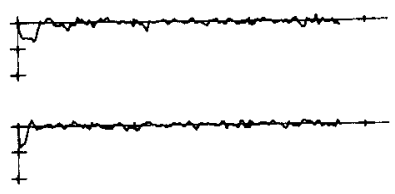

Figure 12. Comparison of singlechannel activity with and without a depolarizing prepulse. Left hand column Groups of 3 records taken during 15 msec voltage steps to the indicated voltage after the standard 300 msec prepulse to -120 or $-140 \mathrm{mV}$ (step records). Right-hand column, Current records taken at the same voltages immediately after a brief conditioning pulse to $+20 \mathrm{mV}$ (tail records). Conditioning pulses were 0.5 to $1 \mathrm{msec}$ in duration. The current records are obtained at the indicated voltages (no voltage transitions occur during the current records). Channel openings are seen immediately after the conditioning pulse. 

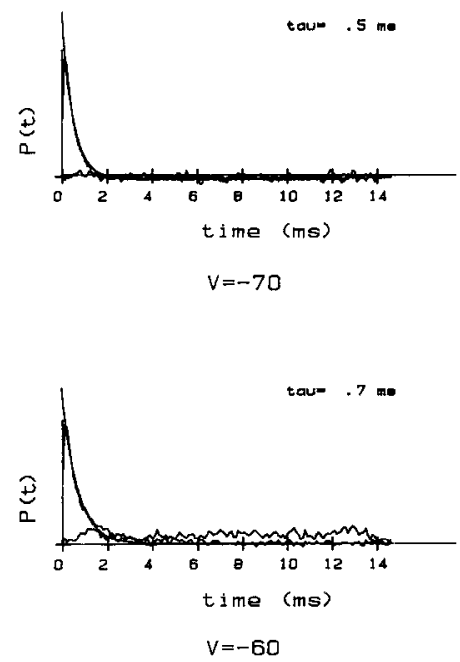

Voltage dependence of sosteps and at the same voltage after a brief depolarized conditioning pulse (tails). Each panel superimposes ensemble averages obtained from records similar to the step and tail records of Figure 12. The amplitudes of the averages have been arbitrarily scaled to facilitate comparison of their kinetics. Tail averages decay quickly and are fitted with single-exponential functions whose mean is the mean duration of the channel openings obtained during both the step and tail experiments (upper valve of $\tau$ ). Step averages have an exponential function fitted to the declining phase $\left(\tau_{h}\right.$, lower value of $\left.\tau\right)$.
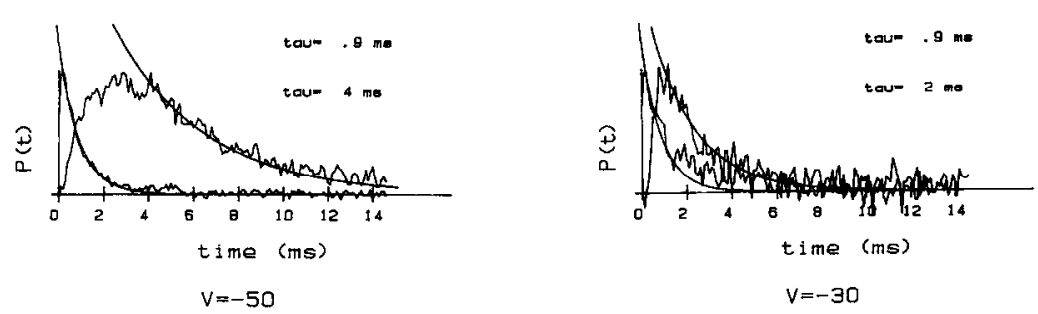

amplitudes have been arbitrarily scaled to facilitate comparison of the kinetics. The tail current records are superimposed with a single exponential whose time constant is the mean duration of all openings during both the step and tail experiments. The step averages are fitted with a single exponential to the falling phase $\left(\tau_{h}\right)$.

A number of conclusions can be drawn from Figure 13. Below the voltage where appreciable activation occurs, the tail currents are well fitted by the exponential predicted from the mean open duration, indicating that the reopenings do not contribute significantly to the tail kinetics. A slow component is seen in the tail averages at higher voltages, where some activation of channels is expected. The voltage dependence of the slow component correlates with the activation curve (Figs. 3, 7) and is the opposite of what would be expected from the voltage dependence of reopening (Fig. 9). This component is similar to the slower components of the step averages and represents, we believe, the late openings of channels that remained in closed (but not inactivated) states during the depolarizing conditioning pulse. The tail averages should be, in fact, the sum of the true tail average (consisting of only channel closings) and the step average at the same voltage, possibly with somewhat faster kinetics due to a perturbation of the distribution of closed states that occurred during the prepulse. The fast component of the tail averages, then, represents the fate of channels after opening (inactivation) and the slow component represents the slow opening process (activation). The tail current experiments allow us to extend the voltage range over which we can measure open durations. There is very little activation in the range -70 to $-60 \mathrm{mV}$, as can be seen from the step averages. Durations measured from tail records in this range are faster than at higher voltages, due to the increase in the closing rate.

The difference in the time courses of the step and tail averages results from different contributions of opening processes to the averages obtained with the 2 techniques. During the step averages the opening process is slow and dominates the overall kinetics, as we have concluded previously. During the tail averages, the opening process becomes negligible and inactivation dominates. Comparison of these 2 averages across the voltage range illustrates the voltage dependence of these 2 processes. At low voltages $(-70$ to $-60 \mathrm{mV}$ ), the probability of opening is low and the opening rate is slow. Openings in this range are brief because the closing (deactivation) rate is comparable to the inactivation rate and increases with voltage as the deactivation rate diminishes. At intermediate voltages, the deactivation rate becomes vanishingly small and the open durations are determined by the inactivation rate, which, as we concluded in the preceding section, has a very weak voltage dependence. In this intermediate voltage range, the overall kinetics are dominated by the slow opening process and the channels tend to open on average 1 time before inactivating (there is some voltage dependence in the contribution of reopenings to the overall kinetics, however; see Fig. 9). The difference between the tail and step averages, then, is due to delayed openings that occur after a step to intermediate voltages. At higher voltages, the opening rates become faster and the declining phase has contributions from both slow opening and inactivation. At even higher voltages, the opening process becomes still faster and the inactivation rate is limiting. This is the voltage range where mean durations and $\tau_{h}$ values converge (see Fig. 5). In many cases, both $\tau_{h}$ and the mean duration remain constant after they converge, although in some cases we have seen them both decrease. This decrease reflects the voltage dependence of open channel inactivation at high voltages that we have indicated earlier.

In summary, then, analysis of channel behavior contributing 
to tail currents suggests that the random process is time homogeneous and Markovian, confirms our previous conclusions, and extends the range of voltages over which channel open time can be measured. The nonexponential decay seen at larger depolarizations is, as expected, the result of continuing activation after the offset of the brief activating prepulse.

\section{Discussion}

In this paper, using some new methods of analysis, we extend our previous results and conclusions to a wider range of voltages. Over the normal activation range, neuroblastoma sodium channels usually open at most once during a depolarizing step and quickly inactivate or, for small depolarizations, deactivate. The open durations are much shorter than $\tau_{h}$, the decay time constant of the average currents. In these neurons, then, the process that might ordinarily be identified as inactivation is an expression of the slow opening (that is, activation) of channels. The mean open time exhibits little voltage dependence. Also, only a slight voltage dependence is found in the probability of a channel reopening calculated from the distribution of openings per voltage step (Aldrich et al., 1983), by the analysis of $p(t)$ and $\hat{p}(t)$ of Figures 8 and 9 or by direct observation during tail current experiments. The absence of appreciable voltage dependence of these values leads to the conclusion that the rate of inactivation of open channels is not very voltage dependent $\left(0.1-0.5 e^{-}\right)$, whereas the deactivation rate is significantly voltage dependent (2.5-3.5 $e^{-}$). The inactivation of closed channels is, however, much more voltage dependent than the inactivation of open channels, with an equivalent gating charge of approximately 3 $e^{-}$(calculated from a Boltzmann distribution; see Aldrich and Stevens, 1983).

Our conclusions for these mammalian sodium channels differ from several models of sodium channel gating that have assumed the inactivation process is uniformly the slowest step (Hodgkin and Huxley, 1952; Gilly and Armstrong, 1982). Additional support for the view presented here comes from pharmacological experiments in mammalian cells that found peak current was significantly increased by agents that remove or slow inactivation (Patlak and Horn, 1982; Gonoi et al., 1984), an observation consistent with a large portion of channel openings occurring after the peak and with overall kinetics being dominated by opening processes.

\section{Are channels independent?}

We have been unable to detect interchannel cooperativity and have concluded that any possible cooperativity present would not have significantly affected our observations. We cannot assert, however, that a channel never influences the behavior of its neighbors. First, we have not placed quantitative limits on the magnitude of possible cooperativity present in our experiments. To do so would require specific models of cooperativity, and we have not examined such models. Second, cooperativity of a sort not tested for might be present. For example, if closed channels influence the behavior of other closed channels, we would not have detected this effect. Finally, channels are quite sparse in our patches-on the order of $1 / \mu \mathrm{m}^{2}-$ and may be present in numbers too small to reveal relatively weak interchannel influences. Horn ct al. (1984) have found similar open times for step and tail currents through $N$-bromoacetamidetreated channels. Cooperativity between individual batrachotoxin-treated channels (Iwasa et al., 1985) and untreated chan- nels (Kiss and Nagy, 1985) has been suggested. We have found no evidence for cooperative behavior but cannot exclude the possibility that it may be present in some circumstances.

\section{Comparison with other single-channel studies}

Although most of our results agree with the observations of others, some differences with published findings are evident. By far the most striking difference between our work on cell-attached patches is with that of other laboratories using isolated patches who report a difference in sodium channel kinetics. Our values of the macroscopic inactivation time constant $\left(\tau_{h}\right)$ obtained from average currents vary from 4 to $1.2 \mathrm{msec}$ over a 30 $\mathrm{mV}$ range at $11^{\circ} \mathrm{C}$ and from 4.3 to $0.4 \mathrm{msec}$ over a $60 \mathrm{mV}$ range at $20^{\circ} \mathrm{C}$ (Aldrich et al., 1983). These values are similar to the time course of average currents from cell-attached patches in myoballs at 18 and $22^{\circ} \mathrm{C}$ (Sigworth and Neher, 1980) and to macroscopic values of $\tau_{h}$ in neuroblastoma cells (Moolenaar and Spector, 1978; Huang et al., 1982; Gonoi et al., 1984), myoballs (Fenwick ct al., 1982), and $\mathrm{GH}_{3}$ cclls (Fernandez et al., 1984). They are significantly faster, however, than values reported in neuroblastoma (Nagy et al., 1983; Quandt and Narahashi, 1984) and $\mathrm{GH}_{3}$ cells (Vandenberg and Horn, 1984) at 6-10 $0^{\circ} \mathrm{C}$. The $\tau_{h}$ values in these studies differ from ours mostly at hyperpolarized voltages, where $\tau_{h}$ is slow, but this is the voltage range over which these authors have carried out their most detailed singlechannel analysis (Quandt and Narahashi, 1982; Nagy et al., 1983; Vandenberg and Horn, 1984). There is general agreement that in this range the open durations increase with voltage (Fenwick et al., 1982; Nagy et al., 1983; Horn et al., 1984), an observation expected if the deactivation rate decreases with depolarization faster than the open channel inactivation rate increases (as we have concluded above). With larger depolarizations, as $\tau_{h}$ decreases, the mean durations remain fairly constant or tend to show a maximum and then decrease. The declining phase of this relationship is difficult to study because of the decrease in the single-channel current with larger voltage steps and the concomitant difficulties in accurate measurements of duration. We have noticed, however, that in patches where there is a decrease in open duration with increasingly positive voltages, this decrease occurs at voltages above that where the mean durations and $\tau_{h}$ curves have converged and the overall gating is rate limited by the inactivation rate.

In parallel with the differences found in macroscopic kinetics between our cell-attached measurements and some whole-cell studies (with concomitant alteration in the internal environment), our mean open durations are much briefer than reported values from some studies on isolated patches. We normally record open durations of $0.5-1 \mathrm{msec}$ over a wide voltage range and with a great deal of consistency from patch to patch. This is similar to or slightly shorter than values reported from cellattached patches on myoballs (Sigworth and Neher, 1980), outside-out patches from chromaffin cells (Fenwick et al., 1982), inside-out patches from neuroblastoma (Quandt and Narahashi, 1982; Horn and Standen, 1983), and inside-out patches from cardiac myocytes (Cachelin et al., 1983; Grant et al., 1983). Other studies, however, using isolated patches, have reported much longer open durations. Horn et al. (1984), Horn and Vandenberg (1984), and Vandenberg and Horn (1984), using $\mathrm{GH}_{3}$ cells at $9^{\circ} \mathrm{C}$, have rarely reported mean open durations less than $2 \mathrm{msec}$, have consistently published values around $4 \mathrm{msec}$, and have even reported mean durations as long as $8.8 \mathrm{msec}$. Nagy 
et al. (1983) have reported means of $1-3.8 \mathrm{msec}$ in neuroblastoma cells, but their open duration histograms have a large slower second exponential component. Since these latter authors used averaged data of patches, it is difficult to know how much of this nonexponential character is a result of heterogeneity among channels.

We have not carried out systematic investigations designed to identify the mechanism underlying the difference in properties of cell-attached and isolated patches. Our shorter durations are not an artifact of measuring open times from overlapping events because we used inactivating prepulses to reduce the probability of a channel opening and therefore the probability of overlaps, and temperature differences of only a few degrees are insufficient to account for the effect. Many of the other studies measured open durations by dividing the integral of current during a trace by the number of closing transitions. This is a useful method for measuring durations in the presence of overlapping opening events but suffers from disadvantages. Because estimates of mean open time are difficult to correct for missed events, the integral method can lead to an overestimate of open duration when many channels are simultaneously open because of the increased probability of missing events with many overlapping openings. The error would be voltage dependent and greater in patches with larger numbers of channels. This could possibly account for the results of Kiss and Nagy (1985), who found a voltage dependence of open durations when measured from overlapping events but not from isolated openings. We do not feel, however, that differences in measuring channel open times can explain the large discrepancy in our open durations and those of Horn and Vandenberg (1984) and Vandenberg and Horn (1984). Since we have observed a lengthening of mean open time in going from cell-attached to isolated patches, we are confident that the difference reflects a modification of the channels in isolated patches, although we do not understand the precise mechanism (see also Horn and Vandenberg, 1986).

Although our open duration distributions are well fitted by single exponentials, we have designed specific statistical tests that reveal an excess of openings longer than would be expected from the mean. Isolated patches have a greater tendency towards excess long openings. Nagy et al. (1983) have reported doubleexponential open time distributions with short time constants similar to ours and longer ones similar to those of Vandenberg and Horn (1984). The possibility that channels are modified upon patch isolation towards longer open times is consistent with a slowing of the open channel inactivation rate, as would be the higher probability of reopening reported by Vandenberg and Horn (1984). It is interesting to note that perfused squid axons have slower macroscopic inactivation and more steadystate current than do intact axons (Meves, 1978). Again, we do not understand the mechanism for this change, although differences in the ionic environment are known to affect the gating of the channel. Both cations (Oxford and Yeh, 1985) and anions (Dani et al., 1983) have been shown to modify sodium channel gating and voltage dependence. Large complex ions in the normal cytoplasmic environment could interact with the channel differently than the fluoride or chloride ions used for isolated patches. Alternatively, posttranslational modifications of the channel such as phosphorylation (Costa et al., 1982) may change with patch isolation. Also, sodium channels might exist in a number of slowly interchangable gating modes with different mean open times (Patlak and Oritz, 1985), or different types of sodium channels may exist (Baud et al., 1982; Matteson and
Armstrong, 1982; Gilly and Armstrong, 1984; Benoit et al., 1985), some of which may preferentially survive in isolated patches.

\section{Voltage dependence of inactivation}

We have concluded that the open channel inactivation rate has very little voltage dependence, with an equivalent gating charge of only a few tenths of an electronic charge. This is in agreement with gating current measurements in squid axons (Armstrong and Bezanilla, 1977; Nonner, 1980; Armstrong, 1981). Stimers et al. (1985) have argued that there must be some voltage dependence to the inactivation mechanism in order to account for the saturation of the peak conductance versus voltage curve. They calculate that an inactivation gating charge of $0.6 e^{-}$is sufficient but do not distinguish between open and closed channel inactivation. It is conceivable that most of the voltage dependence of inactivation could be from the resting to inactivated pathway and that open channel inactivation is relatively voltage independent. This would agree with our findings (Aldrich and Stevens, 1983). It is important to note that our measurements are of inactivation rate and therefore reflect the voltage dependence of the barrier height. Gating currents measure an equilibrium voltage dependence between states and therefore agreement between the 2 methods should not necessarily be expected.

Horn et al. (1984) and Vandenberg and Horn (1984) have found a much greater voltage dependence of open channel inactivation, with as much as $2-3$ equivalent gating charges. The difference between their interpretation and ours may be related to the differences in channel kinetics discussed above. Since their open times are so much longer and the rates so much slower than ours, they may be measuring the rate of a different process (such as slow inactivation that survives in isolated patches) or that of channels in a different mode of gating. It is also possible that they have investigated a voltage range more hyperpolarized than we have and that open channel inactivation is more voltage dependent in this range. In fact, they postulate a saturation of the open channel inactivation rate at higher voltages, as did Hodgkin and Huxley (1952), although their saturating rates are a factor of 2-3 slower than those we have measured.

\section{Limits of our conclusions}

The picture we present is quite different from the standard view derived from squid axons. We must stress that our conclusions may well not apply to squid or other preparations, and only by specific studies can the range of validity be estimated. Since we have made similar observations on other mammalian cell types $\left(\mathrm{GH}_{3}\right.$ and rat myoballs), we do not feel that the neuroblastoma sodium channels are aberrant. In any case, our findings serve as a caution to the temptation of concluding, without singlechannel data, that the macroscopic $\tau_{h}$ must necessarily correspond to the characteristic time for open channel inactivation.

In this paper we have only begun the full analysis of sodium channel behavior required if gating is to be understood at the molecular level. Our strategy has been to isolate part of the system-here we have focused on open channel propertiesthat can be analyzed relatively unambiguously. The challenge for the future will be to extend this analysis to the full range of states to which this interesting and complex protein has access.

\section{Appendix-Terminology}

The terminology used in this paper for time-independent transition probabilities between states differs from 2 previous pub- 
lications (Aldrich and Stevens, 1983; Aldrich et al., 1983). The present symbols are used because they are, in the context of the theory presented here, more mnemonic. The following table is provided to relate this terminology to the earlier publications. For resting $(R)$, open $(O)$, and inactivated $(I)$ states, the probabilities are:

\begin{tabular}{cccc} 
Symbols & This paper & $\begin{array}{c}\text { Aldrich and } \\
\text { Stevens }\end{array}$ & Aldrich et al. \\
\hline$R \rightarrow I$ & $Q$ & $A$ & $1-A$ \\
$O \rightarrow I$ & $F$ & $B$ & $1-B$ \\
$R \rightarrow O$ & $1-Q$ & $1-A$ & $A$ \\
$O \rightarrow R$ & $1-F$ & $1-B$ & $B$
\end{tabular}

\section{References}

Aldrich, R. W., and C. F. Stevens (1983) Inactivation of open and closed sodium channels determined separately. Cold Spring Harbor Symp. Quant. Biol. 48: 147-153.

Aldrich, R. W., D. P. Corey, and C. F. Stevens (1983) A reinterpretation of mammalian sodium channel gating based on single channel recording. Nature 306: 436-441.

Armstrong, C. M. (1981) Sodium channels and gating currents. Physiol. Rev. 61: 644-683.352:653-668.

Armstrong, C. M., and F. Bezanilla (1977) Inactivation of the sodium channel. J. Gen. Physiol. 70: 567-590.

Baud, C., R. T. Kado, and K. Marcher (1982) Sodium channels induced by depolarization of the Xenopus laevis oocyte. Proc. Natl. Acad. Sci. USA 79: 3188-3192.

Benoit, E., A. Corbier, and J.-M. DuBois (1985) Evidence for two transient sodium currents in the frog node of Ranvier. J. Physiol. (Lond.) 361: 339-360.

Cachelin, A. B., J. E. De Pcycr, S. Kokubun, and H. Rcuter (1983) Sodium channels in cultured cardiac cells. J. Physiol. (Lond.) 340: 389-401.

Colquhoun, D., and F. J. Sigworth (1983) Fitting and statistical analysis of single-channel records. In Single-Channel Recording, B. Sakmann and E. Neher, eds., pp. 191-263, Plenum, New York.

Costa, M. R. C., J. E. Casnellie, and W. A. Catterall (1982) Selective phosphorylation of the sodium channel by cAMP-dependent protein kinase. J. Biol. Chem. 257: 7918-7921.

Dani, J. A., J. A. Sanchez, and B. Hille (1983) Na channel gating and Ca electrode response. J. Gen. Physiol. 81: 255-281.

Fenwick, E. M., A. Marty, and E. Neher (1982) Sodium and calcium channels in bovine chromaffin cells. J. Physiol. (Lond.) 331: 599635.

Fernandez, J. M., A. P. Fox, and S. Krasne (1984) Membrane patches and whole-cell membranes: A comparison of electrical properties in rat clonal pituitary (GH3) cells. J. Physiol. (Lond.) 356: 565-585.

Gilly, W. F., and C. M. Armstrong (1982) Slowing of sodium channel opening kinetics in squid axon by extracellular zinc. J. Gen. Physiol. 79: 935-964.

Gilly, W. F., and C. M. Armstrong (1984) Threshold channels-a novel type of sodium channel in squid giant axon. Nature 309: 448450.

Gonoi, T., B. Hille, and W. A. Catterall (1984) Voltage clamp analysis of sodium channels in normal and scorpion toxin-resistant neuroblastoma cells. J. Neurosci. 4: 2836-2842.
Grant, A. O., C. F. Starmer, and H. C. Strauss (1983) Unitary sodium channels in isolated cardiac myocytes of rabbit. Circ. Res. 53: 823829.

Hodgkin, A. L., and A. F. Huxley (1952) A quantitative description of membrane current and its application to conduction and excitation in nerve. J. Physiol. (Lond.) 117: 500-544.

Horn, R., and N. B. Standen (1983) Counting kinetic states: The single channel approach. In The Physiology of Excitable Cells, A. Grinnell and W. Moody, eds., pp. 181-189, Liss, New York.

Horn, R., and C. A. Vandenberg (1984) Statistical properties of single sodium channels. J. Gen. Physiol. 84: 505-534.

Horn, R., and C. A. Vandenberg (1986) Inactivation of single sodium channels. In Ionic Channels in Neural Membranes, J. Ritchie and R. Keynes, eds., pp. 71-83. Liss, New York..

Horn, R., C. A. Vandenberg, and K. Lange (1984) Statistical analysis of single sodium channels: Effects of $N$-bromoacetamide. Biophys. J. 45: 323-335.

Huang, L.-Y. M., M. Moran, and G. Ehrenstein (1982) Batrachotoxin modifies the gating kinetics of sodium channels in internally perfused neuroblastoma cells. Proc. Natl. Acad. Sci. USA 79: 2082-2085.

Iwasa, K., G. Ehrenstein, and N. Moran (1985) Dimers of batrachotoxin-modified sodium channels. Biophys. J. [Abstr.] 47: 191a.

Kiss, T., and K. Nagy (1985) Interaction between sodium channels in mouse neuroblastoma cells. Eur. Biophys. J. 12: 13-18.

Matteson, D. R., and C. M. Armstrong (1982) Evidence for a population of sleepy sodium channels in squid axon at low temperature. J. Gen. Physiol. 79: 739-758.

Meves, H. (1978) Inactivation of the sodium permeability in squid giant nerve fibres. Prog. Biophys. Mol. Biol. 33: 207-230.

Moolenaar, W. H., and I. Spector (1978) Ionic currents in cultured mouse neuroblastoma cells under voltage-clamp conditions. J. Physiol. (Lond.) 278: 265-286.

Nagy, K., T. Kiss, and D. Hof (1983) Single Na channels in mouse neuroblastoma cell membrane: Indication for two open states. Pfluegers Arch. 399: 302-308

Nonner, W. (1980) Relations between the inactivation of sodium channels and the immobilization of gating charge in frog myelinated nerve. J. Physiol. (Lond.) 299: 573-603.

Oxford, G. S., and J.Z. Yeh (1985) Interactions of monovalent cations with sodium channels in squid axon. J. Gen. Physiol. 85: 583-602.

Patlak, J., and R. Horn (1982) Effect of $N$-bromoacetamide on single sodium channel currents in excised membrane patches. J. Gen. Physiol. 79: 333-351.

Patlak, J., and M. Oritz (1985) Slow currents through single sodium channels of the adult rat heart. J. Gen. Physiol. 86: 89-104.

Quandt, F. N., and T. Narahashi (1982) Modification of single Na channels by batrachotoxin. Proc. Natl. Acad. Sci. USA 79: 67326736.

Quandt, F. N., and T. Narahashi (1984) Isolation and kinetic analysis of inward currents in neuroblastoma cells. Neuroscience 13:249-262.

Sigworth, F. J., and E. Neher (1980) Single Na channel currents observed in cultured rat muscle cells. Nature 287: 447-449.

Stimers, J. K., Bezanilla, F., and K. E. Taylor (1985) Sodium channel activation in the squid giant axon. J. Gen. Physiol. 85: 65-82.

Vandenberg, C. A., and R. Horn (1984) Inactivation viewed through single sodium channels. J. Gen. Physiol. 84: 535-564.

Yamamoto, D., F. N. Quandt, and T. Narahashi (1983) Modification of single sodium channels by the insecticide tetramethrin. Brain Res. 274: 344-349. 